\title{
Transparent Montmorillonite/cellulose Nanofibril Nanocomposite Films: the Influence of Exfoliation Degree and Interfacial Interaction
}

\section{Chuan Sun}

South China University of Technology

Guanhui Li

South China University of Technology

Jingyu Wang

South China University of Technology

Zhiqiang Fang ( $\nabla$ mszhqfang@scut.edu.cn )

South China University of Technology

\section{Famei Qin}

South China University of Technology

\section{Kaihuang Chen}

South China University of Technology

Jie Zhou

South China University of Technology

\section{Xueqing Qiu}

Guangdong University of Technology

\section{Research Article}

Keywords: Cellulose nanofibrils, Nanoclays, Layered structure, Nano composites, Interface interactions

Posted Date: January 11th, 2022

DOI: https://doi.org/10.21203/rs.3.rs-1210316/v1

License: (1) This work is licensed under a Creative Commons Attribution 4.0 International License. Read Full License 


\section{Abstract}

To obtain high performance of nanocomposite films made of cellulose nanofibrils (CNFs) and montmorillonites (MMTs), highly ordered nanostructures and abundant interfacial interactions are of extreme importance, especially for CNF film with high MMT content. Here, we tend to unveil the influence of exfoliation degree of MMTs and their interfacial interactions with CNFs on the properties of ensuing nanocomposite films. Monolayer MMTs prefer to form highly ordered nanostructure during water evaporation induced self-assembly. The obtained nanocomposite film with $30 \mathrm{wt} \%$ monolayer MMTs exhibits a tensile strength of $132 \mathrm{MPa}$, a total light transmittance of $90.2 \%(550 \mathrm{~nm})$, and water vapor transmission rate (WVTR) of $41.5 \mathrm{~g} \cdot \mathrm{mm} / \mathrm{m} 2 \cdot$ day, better than the film made of original bulk MMTs and CNFs (30 MPa strength, $60 \%$ transparency, and $78.7 \mathrm{~g} \cdot \mathrm{mm} / \mathrm{m} 2 \cdot$ day WVTR). Moreover, the physical properties (153 MPa strength and $20.9 \mathrm{~g} \cdot \mathrm{mm} / \mathrm{m} 2 \cdot$ day WVTR) of nanocomposite film can be further enhanced by constructing ionic interactions between the monolayer MMT and CNF using $0.5 \mathrm{wt} \%$ cationic polyethylenimine (PEI). However, as the amount of PEI continues to increase, its performance will be deteriorated dramatically because of the disordered orientation of monolayer MMTs. This work could provide an insight into the fabrication of high performance MMT/CNF nanocomposite film for advanced applications.

\section{Introduction}

Thanks to the ideal combination of sustainability, potential inexpensiveness, and tailored physical and chemical properties (e.g. tunable optical and mechanical properties and easy of functionalization),(Zhu et al. 2016; Benítez and Walther 2017; Fang et al. 2019; Zhou et al. 2019; Farooq et al. 2019) transparent cellulose nanofibril (CNF) film has been regarded as a potential green material to partially substitute conventional petroleum-based materials for water treatment, biodegradable food packaging, flexible electronics, and energy conversion. However, to promote the commercial utilizations of transparent CNF films, its original performance (e.g., barrier properties, water resistance) should be further enhanced or novel functions such as fire retardancy should be integrated into films.

The integration of 2D nanomaterials into 1D CNFs is a powerful way to confer their striking and complementary properties to CNF films. Montmorillonites (MMTs) have advantages of layered structure with tunable aspect ratios, thermal stability, nontoxicity, good mechanical and antioxidant properties, which make them a promising functional filler for CNF films. When the content of MMTs reached $25 \mathrm{wt} \%$, MMT/CNF nanocomposite film showed superior barrier and mechanical properties as well as flame retardance.(Sinha Ray and Okamoto 2003; Liu and Berglund 2013; Carosio et al. 2015, 2016) Nevertheless, with the increasing addition of MMTs, a substantial decrease in physical properties (e.g. transparency, mechanical properties) is observed due to the aggregation of MMTs.(Wu et al. 2013)

Nature has long provided numerous inspirations for preparing high performance man-made materials with novel functions by using structural hierarchies that span the atomic, nano-, micro-, to macro-level with accuracy that human technology is yet to be achieved.(Gao et al. 2003; Wang et al. 2017; Peng and 
Cheng 2017; Eder et al. 2018; Huang et al. 2019) Inspired by natural nacre with 95 wt\% calcium carbonate tablets, the fine design principles for highly ordered nanostructures and abundant interfacial interactions are proposed.(Guan and Yu 2020; Guan et al. 2020a, p., b) Subtle in-planar orientation of MMTs lays the foundation for preparing CNF-based film with enhanced properties and novel features. Several strategies have been applied to obtain the ordered structure of MMT/CNF nanocomposite films by self-assembly process: (1) exfoliation of bulk MMTs into monolayer platelets via stirring or sonication;(Liu and Berglund 2013; Medina et al. 2019; Ding et al.) (2) the use of CNF as a dispersant for the exfoliation of MMTs. (Fang et al. 2019; Guanhui et al. 2020; Hosseinpour Feizi and Fatehi 2020; Koshani et al. 2020) For example, Ming et.al prepared a highly transparent and self-extinguishing MMT/CNF nanocomposite film with elaborate lamellar nanostructure using $50 \mathrm{wt} \%$ CNF-dispersed natural monolayer MMTs $(\sim 1 \mathrm{~nm}$ thickness).(Ming et al. 2017)

In addition to the fine construction of ordered nanostructures, the design of interfacial interactions between the components is essential for the properties of final MMT/CNF nanocomposite film. Enhanced interfacial interactions can be realized by the addition of crosslinking agents that interact with both components or surface modifications of MMTs and/or CNFs. Yao et al. improved the interfacial interactions by grafting dopamine (DA) to the surface of CNFs, and the resultant transparent nanocomposite film with $50 \mathrm{wt} \%$ MMTs presented superior barrier and mechanical properties even in humidity environment and water.(Yao et al. 2017) Moreover, Xu et al. used quaternary ammonium modified CNF (Q-CNF) as a raw material to prepare MMT/CNF films with superior physical properties, the quaternary ammonium cations on the surface of Q-CNFs could form strong ionic bonds with anionic MMTs.(Xu et al. 2021) Recently, Liu et. al. used synthetic aminoclay as a functional filler to prepare a strong and transparent clay/CNF nanocomposite film by taking advantage of orderly structure and strong aminoclay-CNF ionic bonds. The synthetic aminoclays with nanometer thickness tend to disperse homogeneously in water and form highly ordered structure in nanocomposite film; while their positive surface charge allowed them to form strong ionic bonds with the negative CNFs.(Liu et al. 2018)

To sum up, endeavors have been directed towards constructing subtle brick-and-mortar nanostructure in MMT/CNF nanocomposite films and/or creating abundant strong MMT-CNF interfacial interactions. However, sporadic attention has been paid to correlating the exfoliation degree of MMTs and their interfacial interactions with CNFs on the physical properties of final nanocomposite film. In this study, we first evaluate the differences in mechanical, optical, barrier, and surface properties of CNF-based nanocomposite films with MMTs with varying layered structures. Moreover, the effect of MMT-CNF interfacial interactions on the physical properties of final film is investigated by quartz crystal microbalance (QCM-D) and atomic force microscope (AFM). This work could provide an insight into preparing high performance MMT/CNF nanocomposite film for advanced applications.

\section{Experimental}

\subsection{Materials}


Hardwood pulp (Hongta paper Co. Ltd, Zhuhai) was used as a raw material for the preparation of carboxylated CNFs by TEMPO oxidization system. MMT powder was purchased from Nanocor Co. Ltd, USA. 2,2,6,6-Tetramethyl piperidine-1-oxy free radical (TEMPO, 98\%, AR) and Polyethylenimine (PEI, M.W. $25,000,50 \%$ aqueous solution) was purchased from Macklin Inc., China. Sodium hypochlorite solution (active chlorine $\geq 7.5 \%, A R$ ) and sodium bromide were purchased from Guangzhou Chemical Reagent Co. Ltd, China, and Damao (Tianjin) Chemical Reagent Co. Ltd, China, respectively.

\subsection{Fabrication and characterization of MMT/CNF nanocomposite films}

CNFs and monolayer MTMs suspension were prepared according to our previous publication. ${ }^{17}$ MTM/CNF nanocomposite films were fabricated by water evaporation induced self-assembly.

The transmittance of the nanocomposite films was recorded on an UV-2600 (Shimazu Co., Japan). Tensile tests were performed on a MST tensile tester (MST, US) with a gauge length of $20 \mathrm{~mm}$ and a strain rate of $0.3 \mathrm{~mm} / \mathrm{min}$. Thermogravimetric analysis (TG-DSC thermogravimetric analyzer, Q500, TA Instruments, USA) was used to determine the mass change of film samples under continuous heating conditions $\left(10 \mathrm{~K} / \mathrm{min}, \mathrm{N}_{2}\right)$. The composite membrane cross section and the front surface structure were characterized by SEM and AFM, respectively. In order to describe the water vapor barrier property of PVA/GO membranes, the cup method was performed to obtain the WVTR of samples, and the more detail of WVTR measurement were shown in supporting information.

\subsection{AFM colloidal probe technique and substrate modification}

The modified CNFs-coating $\mathrm{SiO}_{2}$ were prepared by layer-by-layer self-assembly as follows: $\mathrm{SiO}_{2}$ standard spheres $(23 \mu \mathrm{m})$ were used as base spheres, $\mathrm{CNFs}$ were adhered to the surface of $\mathrm{SiO}_{2}$ by silane coupling agent. Then, the modified spheres were attached on the tipless probe at the end of cantilever by using hot-melt adhesive. (AFM tipless probe (NP-010, Bruker Inc., Germany) with nominal cantilever spring constant of $0.12 \mathrm{~N} / \mathrm{m}$ was used in our experiment.

The force measurement substrate was obtained by layer-by-layer self-assembly of PDAC-MTM on $\mathrm{SiO}_{2}$ substrate, and its morphology and roughness were characterized by AFM imaging. The specific AFM force measurement and calculation details were shown in supporting information.

\subsection{QCM-D measurement}

The PEI reinforcement of adsorption behavior between CNFC and monolayer MTMs was confirmed by using a QCM-D (Q-Sense E1 instrument, Biolin Scientific, Sweden). QCM-D crystal sensor was modified by layer-by-layer self-assembly of PADC and monolayer MTMs/CNFs, and its morphology was characterized by AFM imaging. The specific QCM-D measurement and calculation details were shown in supporting information. 


\section{Results And Discussion}

\subsection{Influence of the exfoliation degree of MMTs}

Three MMTs samples are mixed with CNFs dispersion to prepare nanocomposite films, with the aim of exploring the relationship between the exfoliation of MMTs and the physical properties of final film.

Figure 1a-c shows the AFM images of three MMTs samples used in the preparation of CNF-based nanocomposite films and their corresponding thickness is displayed in the left bottom insets. The original MMTs have a multilayer structure with a thickness of $30-45 \mathrm{~nm}$ and their dispersion in water exhibits a deep turbidity (inset in the upper right corner of Figure 1a). After probe sonification, a relatively transparent appearance is observed for partially exfoliated MMTs dispersion (inset in the upper right corner of Figure 1b), which is due to the existence of monolayer MMTs (as shown in Figure 1b). Through the centrifugation of the partially exfoliated MMTs dispersion, monolayer MMTs with a thickness of about $1 \mathrm{~nm}$ MMTs are obtained and their dispersion in water manifests a more transparent appearance compared to partially exfoliated MMTs dispersion (inset in the upper right corner of Figure 1c).

Figure $1 \mathrm{~d}$-f indicates the production of MMT/CNF nanocomposite films by water evaporation induced self-assembly. Three types of MMTs samples are separately added into CNF dispersion to prepare nanocomposite films of $30 \mathrm{wt} \%$ MMTs and $70 \mathrm{wt} \% \mathrm{CNFs}$, and their corresponding visual appearances are demonstrated in Figure 1g-i. The nanocomposite film with $30 \mathrm{wt} \%$ original multilayer MMTs shows a disordered structure and presents a yellow color yet wrinkling phenomenon (Figure 1g). For a nanocomposite film with partially exfoliated MMTs, it possesses a relatively ordered and flat structure (Figure 1h). In comparison to CNF-based nanocomposite films with original MMTs or partially exfoliated MMTs, the film made of monolayer MMTs and CNFs manifests a high transparency, colorless, and smoothness due to its highly ordered structure (Figure 1i).

In short, the degree of exfoliation on MMTs has a significant influence on the ordered structure of final MMT/CNF nanocomposite films. Monolayer MMTs tend to form highly ordered structure during water evaporation induced self-assembly, which facilitates the enhancement in the physical properties of MMT/CNF nanocomposite films.

We carry out a comparative investigation in mechanical, optical, barrier, and surface properties of nanocomposite film consisting of $70 \mathrm{wt} \% \mathrm{CNFs}$ and $30 \mathrm{wt} \%$ MMTs with varying degrees of exfoliation. As shown in Figure 2, with increasing degree of exfoliation on MMTs, enhanced mechanical, optical, barrier, and surface properties are achieved for MMT/CNF nanocomposite film.

Figure $2 \mathrm{a}$ and $\mathrm{b}$ shows the tensile strength and toughness of nanocomposite films with distinct MMTs samples. The nanocomposite film with original multilayer MMTs demonstrates poor mechanical properties (30MPa tensile strength and $0.24 \mathrm{MJ} / \mathrm{m}^{3}$ toughness). When partially exfoliated MMTs are added into CNF dispersion, nanocomposite film indicates a remarkable improvement in mechanical properties, with a tensile strength of $45 \mathrm{MPa}$ and an average toughness of $0.33 \mathrm{MJ} / \mathrm{m}^{3}$. When all MMTs 
used in nanocomposite film are in the form of individual layers, the resulting CNF-based nanocomposite film presents a tremendous enhancement in tensile strength (132 MPa) and toughness $\left(1.72 \mathrm{MJ} / \mathrm{m}^{3}\right)$.

The light transmission of the as-prepared nanocomposite film also changes with the degree of exfoliation on MMTs. Nanocomposite film with $30 \mathrm{wt} \%$ original MMTs displays a light transmittance of $72.0 \%$ at 550 $\mathrm{nm}$. An optical transparency of $83 \%$ is achieved for nanocomposite film with partially exfoliated MMTs. When monolayer MMTs are added into CNF dispersion, nanocomposite film exhibits a transmittance of $90.3 \%$ in the visible spectrum.

Additionally, the barrier properties of MMT/CNF nanocomposite films are highly dependent on their internal structure. Herein, water vapor transmission rate (WVTR) is used to evaluate the barrier properties of nanocomposite film with $30 \mathrm{wt} \%$ MMTs with different degrees of exfoliation. As shown in Figure $2 \mathrm{~d}$, increasing the degree of exfoliation on MMTs shows a positive effect on the WVTR of final CNF-based nanocomposite film. Nanocomposite films with original MMTs has a WVTR of $78.7 \mathrm{~g} \cdot \mathrm{mm} / \mathrm{m}^{2} \cdot$ day. As the increase of exfoliation degree of MMTs, a remarkable decrease in WVTR is observed, indicating a value of $48.2 \mathrm{~g} \cdot \mathrm{mm} / \mathrm{m}^{2} \cdot$ day. When monolayer MMTs are added into CNF dispersion, a WVTR of only 41.5 $\mathrm{g} \cdot \mathrm{mm} / \mathrm{m}^{2} \cdot$ day is achieved.

The exfoliation degree of MMTs will also affect the surface properties of the MMT/CNF nanocomposite films. In this part, the surface roughness was evaluated by atomic force microscope (AFM). As shown in Figure 2, due to the existence of multilayer MMTs, CNF-based nanocomposite films with original MMTs and partly exfoliated MMTs show a surface roughness of $59.753 \mathrm{~nm}$ (Figure 2e) and $53.705 \mathrm{~nm}$ (Figure $2 \mathrm{f})$, respectively, over a scanning area of $15 \mu \mathrm{m} * 15 \mu \mathrm{m}$. However, a low surface roughness of $\sim 8.0 \mathrm{~nm}$ (Figure $2 \mathrm{~g}$ ) is obtained because of the high in-plane alignment of monolayer MMTs in the internal structure of nanocomposite film. Furthermore, the surface morphologies of CNF-based nanocomposite films with different MMTs samples are characterized by scanning electron microscope (SEM). Rough surface with large blocs is observed for CNF-based nanocomposite film with original bulk MMTs or partially exfoliated MMTs (Figure S1a and b). However, monolayer MMT/CNF nanocomposite film displays a smooth surface without obvious large blocs (as shown in Figure S1c)

Previous works have proved that higher ordered structure of MMT/CNF film would lead to superior physical properties.(Liu and Berglund 2012; Wu et al. 2012; Ho et al. 2012; Kochumalayil et al. 2013) Here, SEM are utilized to analyze the internal structures of CNF-based nanocomposite films with three MMTs samples (30 wt\%). All samples display a closely packed density (Figure 3). Figure 3a shows the crosssectional SEM image of cryo-fractured surface of CNF-based nanocomposite film with $30 \mathrm{wt} \%$ original MMTs. As we can see that layered structure typical for MMT/CNF nanocomposite film is not obvious, whereas large size localized heterogeneities are observed in the internal structure, which is ascribed to the agglomeration of multilayer MMTs that leads to poor mechanical, optical, barrier, and surface properties.

For CNF-based nanocomposite film with partially exfoliated MMTs, its degree of structural ordering improves significantly, and no large size localized heterogeneities is observed throughout the thickness of 
the film (Figure 3b). However, due to the existence of multilayer MMTs, typical layered structure is still not obvious and only slight enhancements in tensile strength, toughness, light transmittance, and surface smoothness are achieved.

Monolayer MMTs can uniformly embed in the fibril network of CNFs and tend to form highly ordered nanostructure during water evaporation induced self-assembly. Figure $3 \mathrm{c}$ shows the typical layered structure obtained from the intercalated monolayer MMTs with CNFs. The high in-plane stacking of both monolayer MTMs and CNFs in the internal structure is conducive to the dissipation of applied stress, the propagation of light, and the block of gas diffusion, thus contributing to the remarkable enhancement in physical properties of final nanocomposite films.

To further understand the effect of internal structure on the mechanical properties, the tensile-broken surfaces of CNF-based nanocomposite films with three MMTs samples are analyzed. MMT agglomerations with different sizes are observed in the tensile-broken surfaces of CNF-based nanocomposite films with original bulk MMTs (Figures 3d, e) or partially exfoliated MMTs (Figures $3 f, g$ ). The disordered stacking of bulk MMTs in the internal structure of nanocomposite film induces defective internal structure that hinders the dissipation of stress during tensile testing. The upper strain limit of the plateaus for both nanocomposite films is lower than 1.25\% (Figures 2a). As shown in Figure $3 \mathrm{~h}$ and $\mathbf{i}$, an obvious pull out of MMTs and CNFs is observed in the tensile fracture surface due to the homogeneous intercalation structure. The regular layered structure increases the contact areas between the MMTs and the CNFs, thereby improving the stress and strain of nanocomposite film (Figure 3j).

In sum, the highly ordered structure plays a pivotal role in mechanical, optical, barrier, and surface properties of MMT/CNF nanocomposite film. Reducing the numbers of layers of bulk MMTs is highly desired for high performance MMT/CNF film. Monolayer MMTs are inclined to form highly ordered structure in CNF-based nanocomposite film, which facilitates the propagation of visible light, the effective transfer of applied stress, the prevention of gas diffusion, as well as reduced surface roughness. The resulting CNF-based nanocomposite film with monolayer MMTs presents a remarkable enhancement in physical properties, with a transparency of $90.5 \%$ at $550 \mathrm{~nm}$, a tensile strength of $132 \mathrm{MPa}$, and a WVTR of $41.5 \mathrm{~g} * \mathrm{~mm} / \mathrm{m}^{2 *}$ day.

\subsection{Influence of interfacial interactions between CNF and monolayer MMT}

An orderly internal structure lays the foundation for fabricating MMT/CNF nanocomposite films with enhanced properties.(Peng and Cheng 2017, p.) Besides, the interfacial interactions between CNFs and MMTs can govern and promote their physical properties. Polyethylenimine (PEI) is a wet strength agent widely used in paper industry and has a strong affinity for cellulose fibers due to its high positive charge density. 
In this work, PEl is employed to improve the interfacial interactions between the MMTs and CNFs by introducing ionic bonding (Figure 4a). The whole procedure of preparing monolayer MMT/CNF nanocomposite film with PEI is shown in Figure S2. Well-dispersed monolayer MMTs are first mixed with CNFs to obtain aqueous MMT/CNF mixture, and PEl is then added dropwise into above suspension under magnetic stirring at a speed of $1000 \mathrm{rpm} / \mathrm{min}$. Finally, MMT/CNF nanocomposite film is obtained by water evaporation induced self-assembly. Figure $4 \mathrm{~b}$ and $\mathrm{c}$ shows the digital images of nanocomposite films with $0 \mathrm{wt} \%$ and $0.5 \mathrm{wt} \% \mathrm{PEI}$, respectively. As we can see that there is no difference in visual appearance of nanocomposite films with/without PEI. However, when the addition of PEl reaches $1 \mathrm{wt} \%$, the nanocomposite film displays a yellow coloration (Figure S3).

The physical properties of MMT/CNF nanocomposite films with varying dosages of PEl are investigated and the results are shown in Figure 5. Both the tensile strength and toughness of the nanocomposite film first rise, and then decrease with the increase of PEl addition from 0 to $1 \mathrm{wt} \%$ based on the total mass of MMTs and CNFs (Figure $5 \mathrm{a}$ and $\mathrm{b}$ ). When the PEI reaches $0.5 \%$, an increase in tensile strength (152 MPa) and toughness $\left(2.61 \mathrm{MJ} / \mathrm{m}^{3}\right)$ is observed as compared to PEl-free nanocomposite film with a tensile strength of $135 \mathrm{MPa}$ and a toughness of $2.22 \mathrm{MJ} / \mathrm{m}^{3}$. As the addition of PEl continues to increase to 1 $w t \%$, nanocomposite film demonstrates a decrease in the tensile strength (113 MPa) and toughness (1.13 $\left.\mathrm{MJ} / \mathrm{m}^{3}\right)$.

The addition of PEI has a negative impact on optical transparency of nanocomposite film when the spectrum is lower than $550 \mathrm{~nm}$. As shown in Figure 5c, with the increase of PEl from $0-1 \%$, the light transmittance curves of nanocomposite films with different PEI dosages nearly overlap in the spectrum of 550-700 nm, however, the optical transparency decreases sharply when the spectrum is in the range of $300 \mathrm{~nm}$ to $550 \mathrm{~nm}$. Note that ultraviolet light cannot pass through the nanocomposite film with $1 \mathrm{wt} \%$ PEI.

PEl can reduce the WVTR of the nanocomposite film. Figure $5 \mathrm{~d}$ displays the WVTR of the nanocomposite films with different dosages of PEI, which indicates a similar phenomenon compared to the tensile strength and toughness. When the PEl addition reaches $0.5 \%$, the nanocomposite film shows a minimum WVTR with a value of $20.9 \mathrm{~g} \mathrm{~mm}^{\star} \mathrm{m}^{2 \star}$ day, which is much lower than PEl-free nanocomposite film (41.5 $\mathrm{g}^{\star} \mathrm{mm} / \mathrm{m}^{2 \star}$ day $)$. As the PEI increases to $1 \mathrm{wt} \%$, there is a minor increase in WVTR $\left(22.1 \mathrm{~g} * \mathrm{~mm} / \mathrm{m}^{2 \star}\right.$ day $)$.

To explore the reinforcing mechanism of PEI for MMT/CNF nanocomposite film, the interfacial interactions between individual components are determined by (QCM-D) and AFM. QCM-D technique is adopted to quantitatively evaluate the intensity of interfacial interactions between MMT and CNF as well as between MMT and PEl adsorbed CNF. As shown in Figure 6a, a CNF film is first coated on the surface of a QCM-D chip, and the adsorption of MMTs by CNF or PEl-adsorbed CNF is then measured (Figure 6b). The adsorption behaviors between CNFs and between MMTs are investigated using same operation procedure (as shown in Figure $6 \mathrm{c}$ and d). 
The change in frequency of QCM-D chip is labeled as $\triangle F$ that reflects the intensity of interfacial interaction between the two components. We can see from Figure $6 \mathrm{~b}$ that $\triangle F_{3}$ (the change in frequency with $P E I$ ) is much higher than $\triangle F_{2}$ (the change in frequency without $\left.P E I\right)$, suggesting the significant increase in adsorption between the MMT and CNF because of the introduction of PEl. Besides, the ratio of $\triangle F_{3}$ to $\triangle F_{2}$ is used to evaluate the enhancement effect of $P E I$ on the interfacial interactions between MMT and CNF, between MMTs, and between CNFs, which shows a value of $3.15,4.61$, and 16.9, respectively. It is worth noting that the ratio $\left(\triangle F_{3} / \triangle F_{2}\right)$ between the CNFs has the largest value among three samples, though a maximum value for $\triangle F_{3}$ between the CNF and MTM is obtained. This is due to the strong electrostatic repulsion between the negatively charged CNFs that leads to a much lower $\triangle F_{2}$ as compared to the other two samples. Therefore, we can conclude that the addition of PEI can produce a strongest interfacial interaction between the MTM and CNF and has the largest enhancement effect on the adsorption between the CNFs.

Additionally, AFM force measurement with colloidal probe technique is applied to determine the interfacial interactions between CNF and MMT in water and $0.1 \%$ PEI solution from molecular point of view. Figure $6 \mathrm{e}$ is the schematic diagram of AFM force measurement which includes a colloidal sphere probe decorated with a thin layer of silane coupling agent and CNFs in turn, and a substrate coated with monolayer MMTs. Then, force/distance (F/D) curves between CNFs and MMTs are measured in water and aqueous PEl solution.

Furthermore, classic Derjaguin-Landau-Verwey-Overbeek (DLVO) theory has been regarded as a powerful tool to explain the electrostatic force and van der Waals force existing in the system. Hence, the approaching F/D curves are fitted by DLVO theory according to our previous work.(Sun et al. 2019) As shown in Figure 6f, there is a strong electrostatic repulsion between the CNFs and the MMTs in pure water. When the AFM force measurement performs in aqueous PEI solution, the interfacial interaction gradually changes from electrostatical repulsion to attractive force, which suggests that PEI can serve as a cationic polymer to effectively enhance the electrostatic attractive interaction between the CNFs and the MMTs.

The physical properties of MMT/CNF nanocomposite film should be enhanced with the increasing dosage of PEI, but opposite result is obtained. Hence, the internal nanostructures of MMT/CNF nanocomposite films with different dosages of PEI are further analyzed. The nanocomposite film without PEI has the lowest roughness of $9.831 \mathrm{~nm}$ (Figure 7a) due to its highly ordered nanostructure (Figure 7d). When $0.5 \mathrm{wt} \% \mathrm{PEl}$ is introduced into MMT/CNF nanocomposite film, there is a minor increase in surface roughness (Figure 7b). As we can see from the SEM image in Figure 7e that the inside layered nanostructure is almost retained. However, the surface roughness dramatically increases to $33.502 \mathrm{~nm}$ as the dosage of PEl increases to $1 \mathrm{wt} \%$ (Figure 7c), which is ascribed to the strong attractive interactions between the MMT and the CNF, between the CNFs, and the MMTs that deteriorate the ordered structure of the nanocomposite film. In comparison to nanocomposite film without PEI, the internal structure of the nanocomposite film with $1 \mathrm{wt} \% \mathrm{PEI}$ is more disordered (Figure 7f). In short, PEl could not only effectively 
enhance the attractive force between the MMT and the CNF, between the CNFs, and the MMTs, but also largely maintain the ordered structure of MMT/CNF nanocomposite film if its dosage is appropriate. Its physical properties will be deteriorated if the amount of PEI continues to increase due to the occurrence of disordered structure.

\section{Conclusion}

In conclusion, we explore the influence of the exfoliation degree of MMT and its interactions with CNF on the physical properties of MMT/CNF nanocomposite film. Complete exfoliation of bulk MMTs facilitates the formation of highly ordered structure in the CNF-based nanocomposite film during water evaporation induced self-assembly, thus significantly contributing to the enhanced physical properties of final MMT/CNF nanocomposite film. The CNF-based nanocomposite film with $30 \mathrm{wt} \%$ monolayer MMTs presents excellent physical properties with an optical transparency of $90.2 \%$ at $550 \mathrm{~nm}$, a tensile strength of $132 \mathrm{MPa}$, a toughness of $1.72 \mathrm{MJ} / \mathrm{m}^{3}$, a surface roughness of $\sim 8.0 \mathrm{~nm}$, and a WVTR of 41.5 $\mathrm{g}^{\star} \mathrm{mm} / \mathrm{m}^{2 *}$ day.

Appropriate amount of PEI could improve the interfacial interactions between MMT and CNFs by introducing ionic bonding. The monolayer MMT/CNF nanocomposite film with 0.5 wt\% PEl exhibits enhanced mechanical and barrier properties, with a tensile strength of 153MPa, a toughness of 2.61 $\mathrm{MJ} / \mathrm{m}^{3}$, and a WVTR of $22.5 \mathrm{~g} * \mathrm{~mm} / \mathrm{m}^{2 \star}$ days. However, a slight deterioration in transparency and surface roughness is observed. As the dosage of PEI continues to increase, physical properties of final nanocomposite film will be dramatically deteriorated due to the disordered orientation of monolayer MMTs. Therefore, the enhancement in interfacial interactions between monolayer MMTs and CNFs cannot be at the expense of ordered structure. This work provides an insight into the fabrication of high performance MMT/CNF nanocomposite film for advanced applications (e.g., flexible electronics).

\section{Declarations}

\section{Acknowledgement}

We would like to appreciate the Fund of the National Natural Science Foundation of China [21978103], the Natural Science Foundation of Guangdong Province [2020B1515020021], and the Fundamental Research Funds for the Central Universities [2019MS083].

\section{References}

Benítez AJ, Walther A (2017) Cellulose nanofibril nanopapers and bioinspired nanocomposites: a review to understand the mechanical property space. Journal of Materials Chemistry A 5:16003-16024. https://doi.org/10.1039/C7TA02006F

Carosio F, Kochumalayil J, Cuttica F, et al (2015) Oriented Clay Nanopaper from Biobased ComponentsMechanisms for Superior Fire Protection Properties. ACS Appl Mater Interfaces 7:5847-5856. 
Carosio F, Kochumalayil J, Fina A, Berglund LA (2016) Extreme Thermal Shielding Effects in Nanopaper Based on Multilayers of Aligned Clay Nanoplatelets in Cellulose Nanofiber Matrix. Advanced Materials Interfaces 3:1600551. https://doi.org/10.1002/admi.201600551

Ding F, Liu J, Zeng S, et al Biomimetic nanocoatings with exceptional mechanical, barrier, and flameretardant properties from large-scale one-step coassembly. Science Advances 3:e1701212. https://doi.org/10.1126/sciadv.1701212

Eder M, Amini S, Fratzl P (2018) Biological composites-complex structures for functional diversity. Science 362:543-547. https://doi.org/10.1126/science.aat8297

Fang ZQ, Hou GY, Chen CJ, Hu LB (2019) Nanocellulose-based films and their emerging applications. Current Opinion in Solid State and Materials Science 23:100764.

https://doi.org/10.1016/j.cossms.2019.07.003

Farooq M, Zou T, Riviere G, et al (2019) Strong, Ductile, and Waterproof Cellulose Nanofibril Composite Films with Colloidal Lignin Particles. Biomacromolecules 20:693-704.

https://doi.org/10.1021/acs.biomac.8b01364

Gao H, Ji B, Jäger IL, et al (2003) Materials become insensitive to flaws at nanoscale: Lessons from nature. PNAS 100:5597-5600. https://doi.org/10.1073/pnas.0631609100

Guan Q-F, Ling Z-C, Han Z-M, et al (2020a) Ultra-Strong, Ultra-Tough, Transparent, and Sustainable Nanocomposite Films for Plastic Substitute. Matter 3:1308-1317.

https://doi.org/10.1016/j.matt.2020.07.014

Guan Q-F, Yang H-B, Han Z-M, et al (2020b) An all-natural bioinspired structural material for plastic replacement. Nat Commun 11:5401. https://doi.org/10.1038/s41467-020-19174-1

Guan Q-F, Yu S-H (2020) A superspreading layering process enabled high performance layered nanocomposites. Sci China Chem 63:873-874. https://doi.org/10.1007/s11426-020-9751-6

Guanhui L, Gaoyuan H, Hong X, et al (2020) Optical and Mechanical Properties of CMCNF-dispersed MMT/CMC-Na Nanocomposite Films: Influence of the Degree of Substitution of CMCNFs. Paper and Biomaterials 18-26. https://doi.org/10.12103/j.issn.2096-2355.2020.04.003

Ho TTT, Zimmermann T, Ohr S, Caseri WR (2012) Composites of Cationic Nanofibrillated Cellulose and Layered Silicates: Water Vapor Barrier and Mechanical Properties. ACS Appl Mater Interfaces 4:48324840. https://doi.org/10.1021/am3011737

Hosseinpour Feizi Z, Fatehi P (2020) Carboxymethylated cellulose nanocrystals as clay suspension dispersants: effect of size and surface functional groups. Cellulose 27:3759-3772. 
https://doi.org/10.1007/s10570-020-03024-w

Huang W, Restrepo D, Jung J-Y, et al (2019) Multiscale Toughening Mechanisms in Biological Materials and Bioinspired Designs. Advanced Materials 31:1901561. https://doi.org/10.1002/adma.201901561

Kochumalayil JJ, Morimune S, Nishino T, et al (2013) Nacre-Mimetic Clay/Xyloglucan

Bionanocomposites: A Chemical Modification Route for Hygromechanical Performance at High Humidity. Biomacromolecules 14:3842-3849. https://doi.org/10.1021/bm400883e

Koshani R, Tavakolian M, Ven TGM van de (2020) Cellulose-based dispersants and flocculants. J Mater Chem B 8:10502-10526. https://doi.org/10.1039/D0TB02021D

Liu A, Berglund LA (2013) Fire-retardant and ductile clay nanopaper biocomposites based on montmorrilonite in matrix of cellulose nanofibers and carboxymethyl cellulose. European Polymer Journal 49:940-949. https://doi.org/10.1016/j.eurpolymj.2012.12.017

Liu A, Berglund LA (2012) Clay nanopaper composites of nacre-like structure based on montmorrilonite and cellulose nanofibers-Improvements due to chitosan addition. Carbohydrate Polymers 87:53-60. https://doi.org/10.1016/j.carbpol.2011.07.019

Liu Y, Yu S, Bergström L (2018) Transparent and Flexible Nacre-Like Hybrid Films of Aminoclays and Carboxylated Cellulose Nanofibrils. Adv Funct Mater 28:. https://doi.org/10.1002/adfm.201703277

Medina L, Nishiyama Y, Daicho K, et al (2019) Nanostructure and Properties of Nacre-Inspired Clay/Cellulose Nanocomposites-Synchrotron X-ray Scattering Analysis. Macromolecules 52:3131-3140. https://doi.org/10.1021/acs.macromol.9b00333

Ming S, Chen G, He J, et al (2017) Highly Transparent and Self-Extinguishing Nanofibrillated CelluloseMonolayer Clay Nanoplatelet Hybrid Films. Langmuir 33:8455-8462.

https://doi.org/10.1021/acs.langmuir.7b01665

Peng J, Cheng Q (2017) High-Performance Nanocomposites Inspired by Nature. Adv Mater 29:. https://doi.org/10.1002/adma.201702959

Sinha Ray S, Okamoto M (2003) Polymer/layered silicate nanocomposites: a review from preparation to processing. Progress in Polymer Science 28:1539-1641.

https://doi.org/10.1016/j.progpolymsci.2003.08.002

Sun C, Fang Z, Qin F, et al (2019) Insight into the Dispersive Mechanism of Carboxylated Nanofibrilllated Cellulose for Individual Montmorillonite in Water. Composites Part B: Engineering 177:.

https://doi.org/10.1016/j.compositesb.2019.107399

Wang L, Chen D, Jiang K, Shen G (2017) New insights and perspectives into biological materials for flexible electronics. Chemical Society Reviews 46:6764-6815. https://doi.org/10.1039/C7CS00278E 
Wu C-N, Saito T, Fujisawa S, et al (2012) Ultrastrong and High Gas-Barrier Nanocellulose/Clay-Layered Composites. Biomacromolecules 13:1927-1932. https://doi.org/10.1021/bm300465d

Wu C-N, Yang Q, Takeuchi M, et al (2013) Highly tough and transparent layered composites of nanocellulose and synthetic silicate. Nanoscale 6:392-399. https://doi.org/10.1039/C3NR04102F

Xu D, Wang S, Berglund LA, Zhou Q (2021) Surface Charges Control the Structure and Properties of Layered Nanocomposite of Cellulose Nanofibrils and Clay Platelets. ACS Appl Mater Interfaces 13:44634472. https://doi.org/10.1021/acsami.0c18594

Yao K, Huang S, Tang H, et al (2017) Bioinspired Interface Engineering for Moisture Resistance in NacreMimetic Cellulose Nanofibrils/Clay Nanocomposites. ACS Appl Mater Interfaces 9:20169-20178. https://doi.org/10.1021/acsami.7b02177

Zhou Y, Chen C, Zhang X, et al (2019) Decoupling Ionic and Electronic Pathways in Low-Dimensional Hybrid Conductors. Journal of the American Chemical Society 141:17830-17837.

https://doi.org/10.1021/jacs.9b09009

Zhu HL, Luo W, Ciesielski PN, et al (2016) Wood-Derived Materials for Green Electronics, Biological Devices, and Energy Applications. Chem Rev 116:9305-9374.

https://doi.org/10.1021/acs.chemrev.6b00225

\section{Figures}




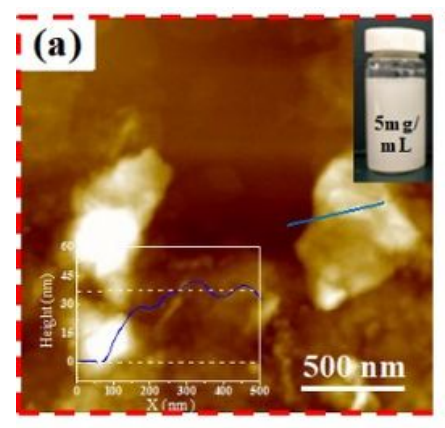

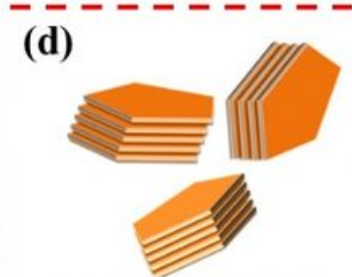

Multilayer MMTs

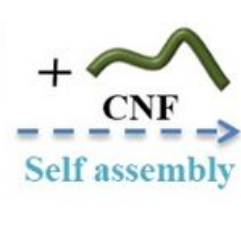

Probe sonication

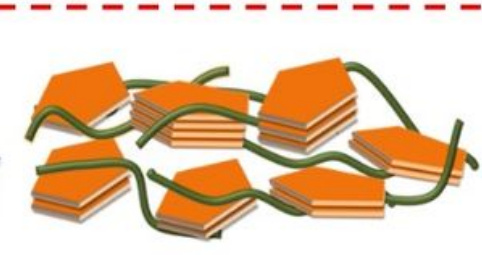

Random structure

(g)

ty University Unit

ty UniversityUnil

ty UniversityUni;

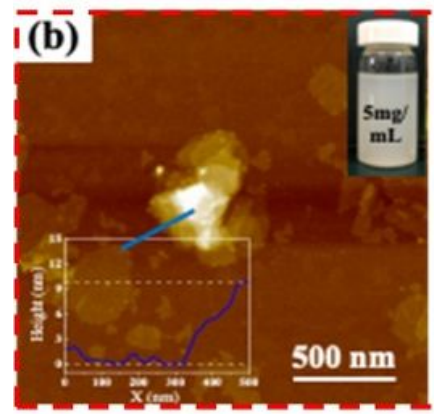

(e)

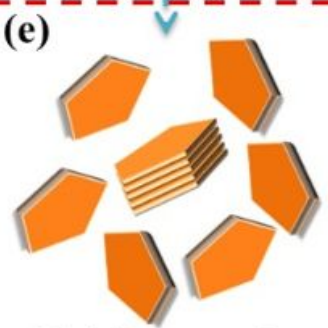

Multilayer and monolayer

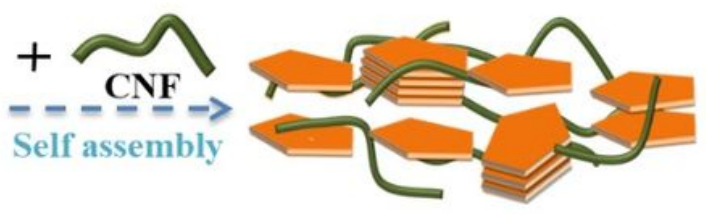

(h) University Univi ty UniversityUniv! ty UniversityUnivi

Random and ordered structure
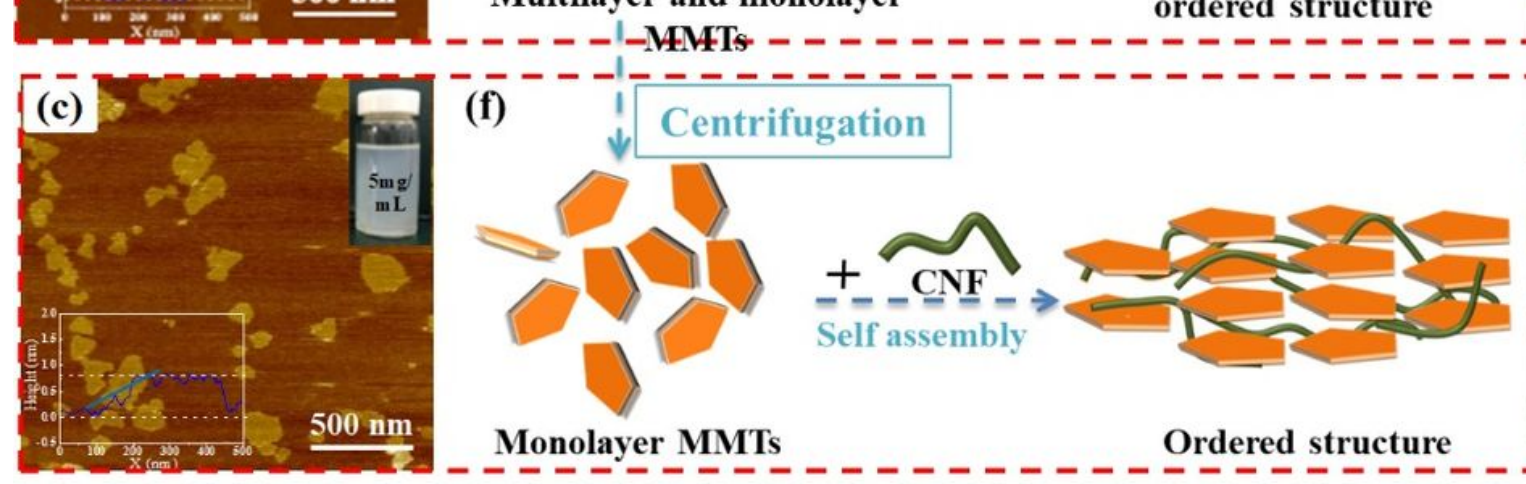

Monolayer MMTs

Ordered structure

(i)

y UniversityUnive

y University Unive

y UniversityUniva

\section{Figure 1}

AFM images of (a) bulk MMTs, (b) partially exfoliated MMTs consisting of multilayer and monolayer MMTs, and (c) monolayer MMTs. Insets in left bottom represent the thickness of three types of MMTs samples while insets in upper right corner indicate the visual appearance of corresponding MMT suspensions. Schematic illustration of the production route for CNF-based nanocomposite films with 30 wt\% (d) original MMTs, (e) partially exfoliated MMTs, or (f) completely exfoliated MMTs (also named monolayer MMTs) and their corresponding digital photos (g-i). 

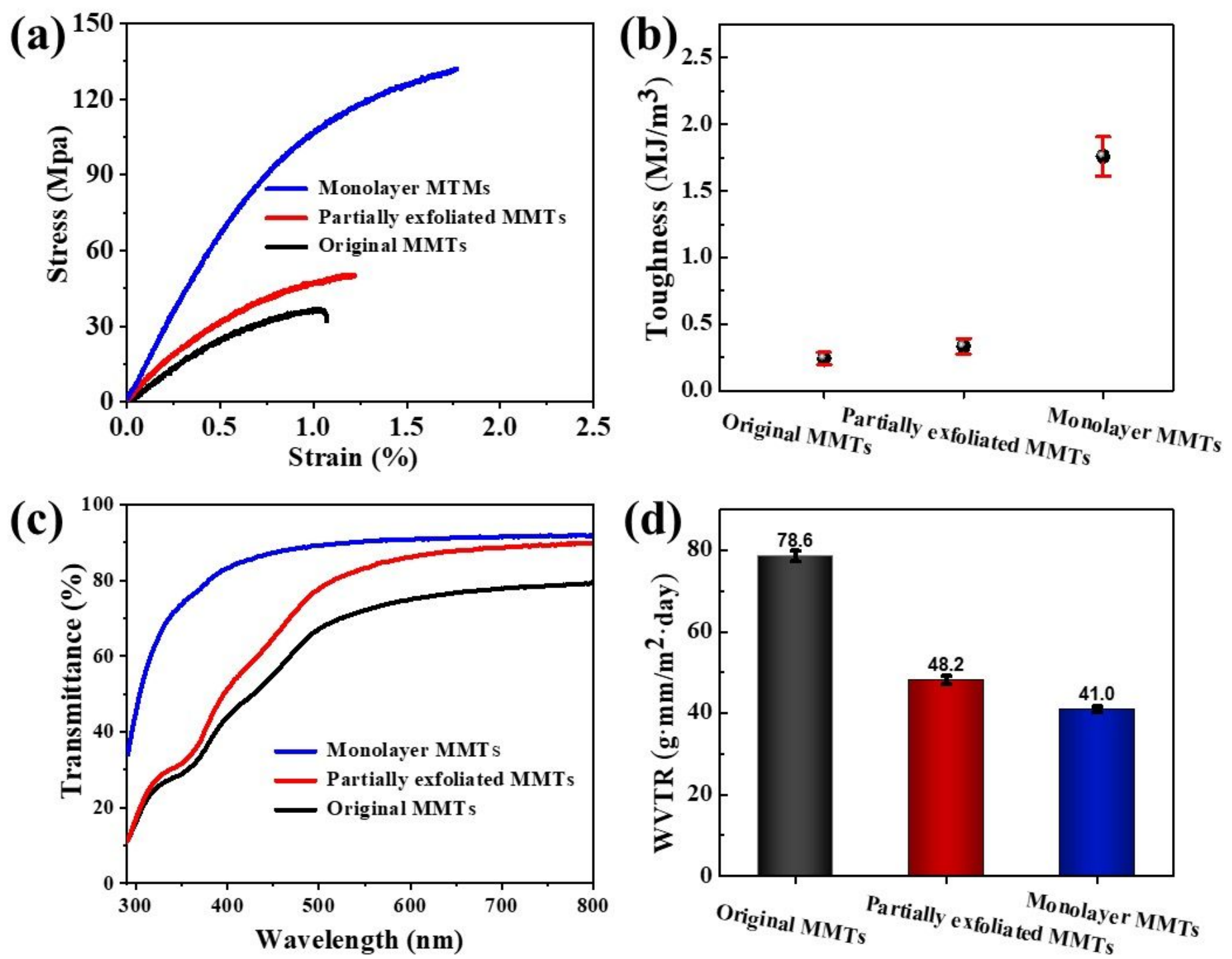

(e)

(f)
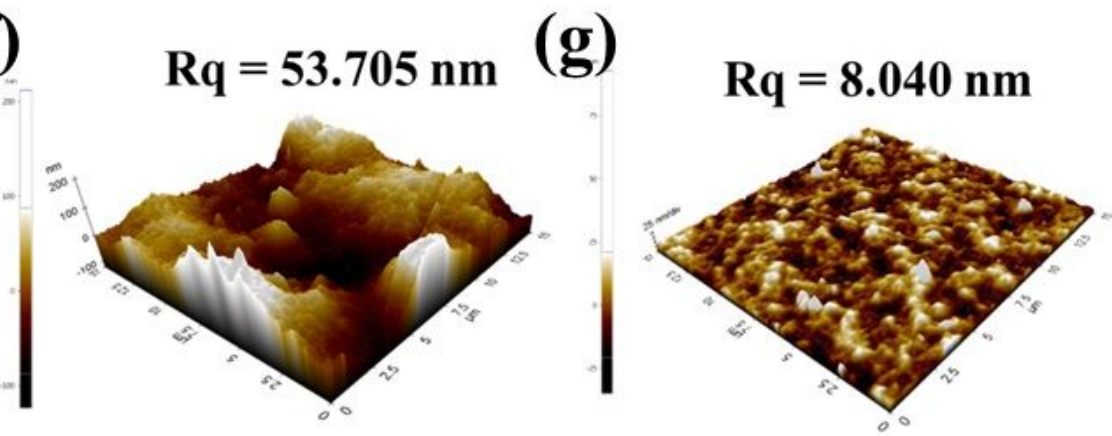

Figure 2

Mechanical, optical, and barrier properties of nanocomposite films made of $70 \mathrm{wt} \% \mathrm{CNFs}$ and $30 \mathrm{wt} \%$ MMTs with different degrees of exfoliation. (a) Stress-strain curve, (b) toughness, (c) light transmission, (d) WVTR. Note that the WVTR is measured under the condition of $39^{\circ} \mathrm{C}$ and $90 \mathrm{RH} \%$. Surface roughness of CNF-based nanocomposite film with different MMTs samples: (e) original MMTs, (f) partially exfoliated MMTs, and (g) monolayer MMTs. 


\section{Figure 3}

Cross-sectional SEM images showing the cryo-fractured surfaces of nanocomposite film consisting of 70 wt\% CNFs and $30 \mathrm{wt} \%$ MMTs with different degrees of exfoliation: (a) original MMTs, (b) partially exfoliated MMTs, and (c) monolayer MMTs. Note that red circle indicates large size localized heterogeneity in the CNF-based nanocomposite film with original MMTs. SEM images showing the tensile broken surfaces of CNF-based nanocomposite films with different MMTs samples: (d, e) original MMTs, $(f, g)$ partially exfoliated MMTs, $(\mathrm{h}, \mathrm{i})$ monolayer MMTs. (j) Schematic diagram of tensile fracture mechanism for monolayer MMT/CNF nanocomposite film.

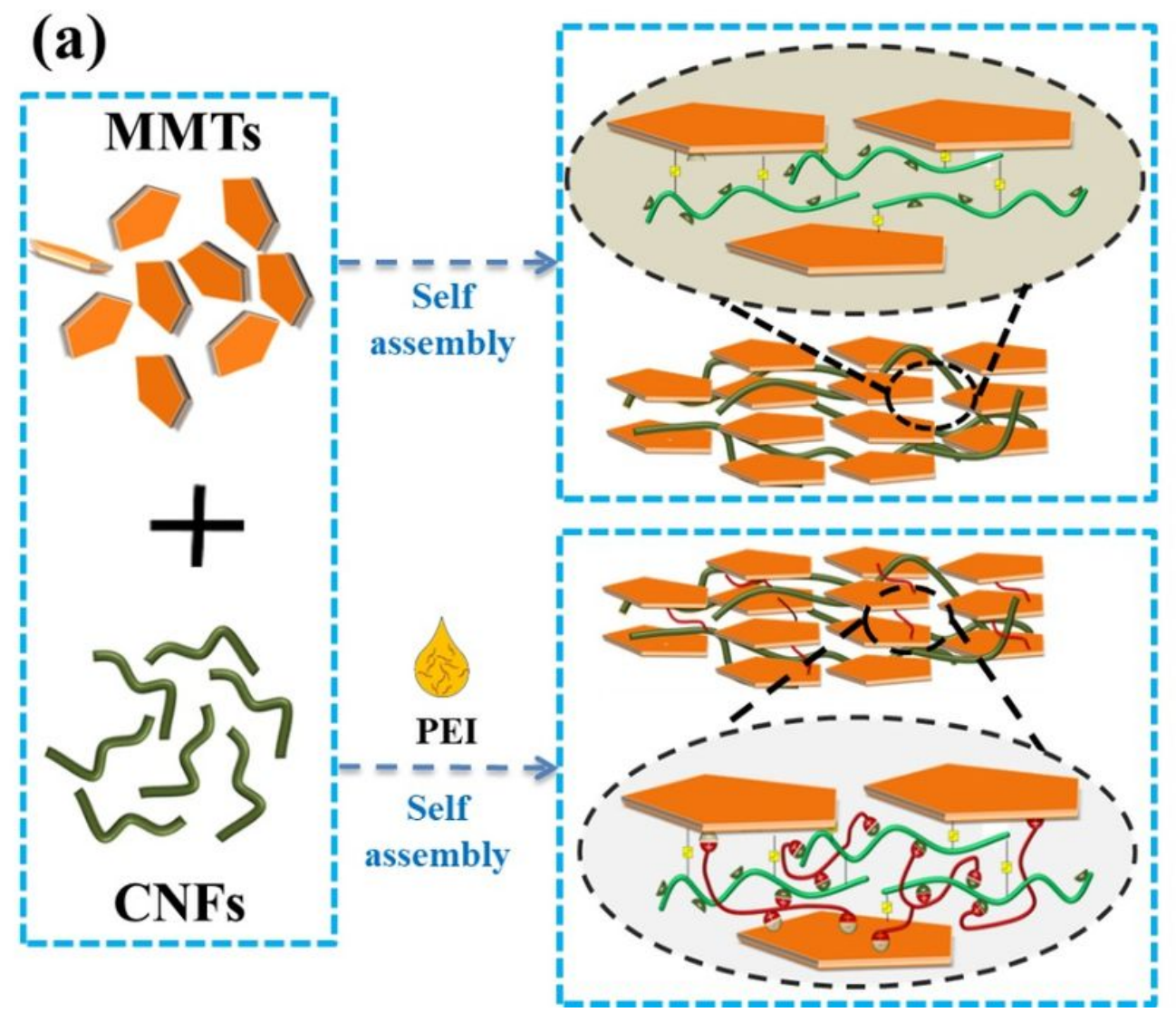

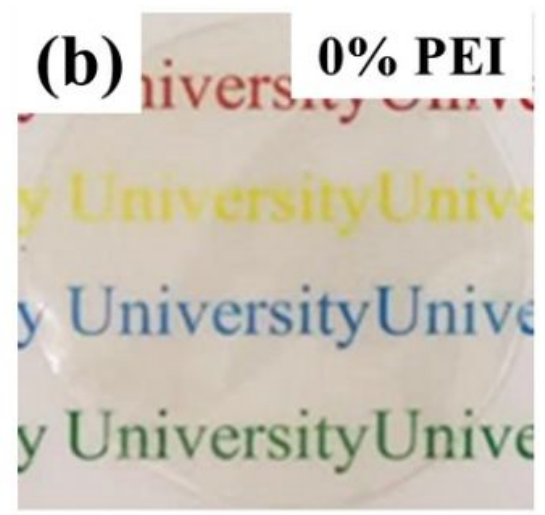

(c) ityl $0.5 \%$ PEI iversityUniversity I iversityUniversity L iversityUniversity I

\section{Figure 4}

(a) Schematic diagram of interfacial interactions between monolayer MMTs and CNFs enhanced with/without PEI. (b) Visual appearance of MMT/CNF nanocomposite films with PEl: (b) $0 \%$, (c) $0.5 \%$. Note that the addition of monolayer MMTs is $30 \mathrm{wt} \%$. 
(a)

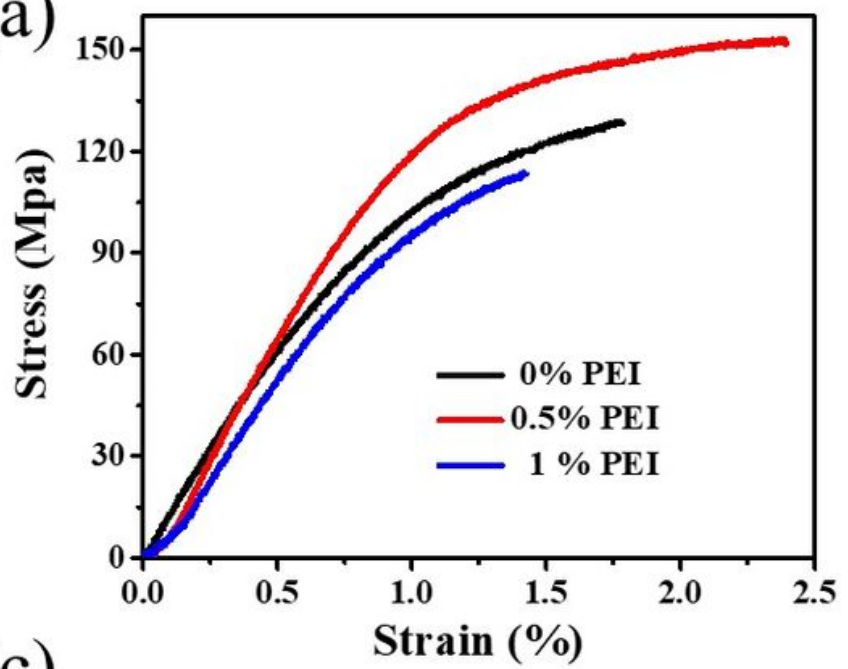

(c)

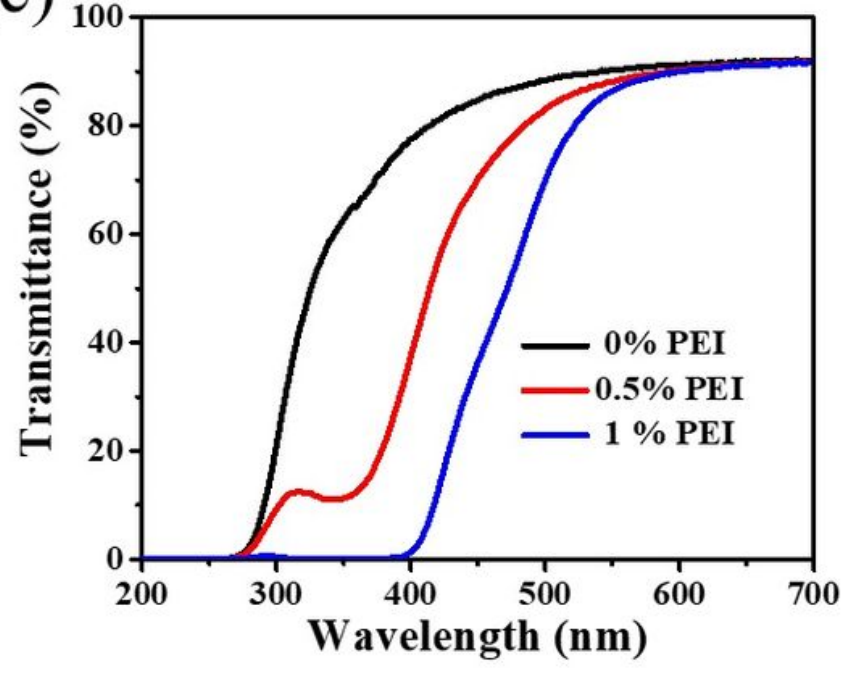

(b) 3

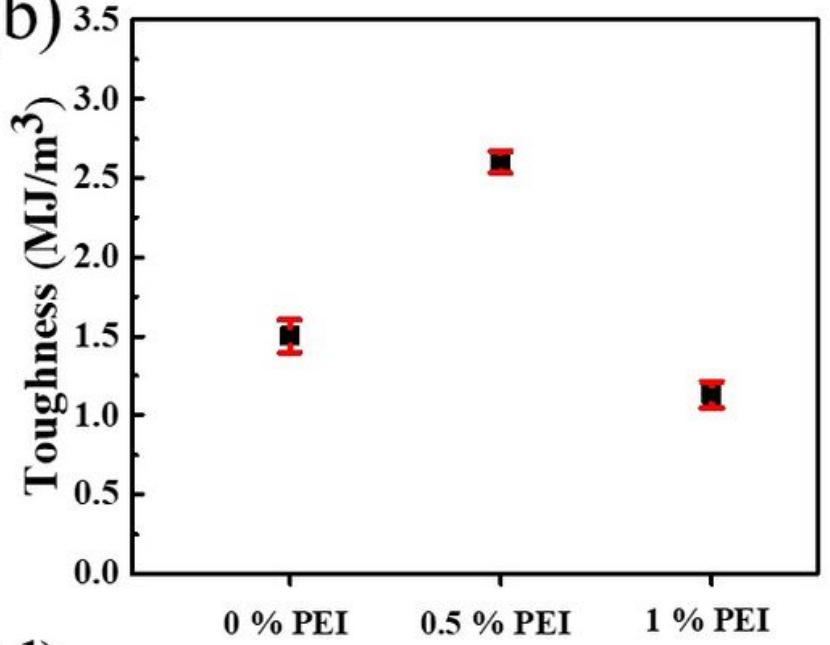

(d)

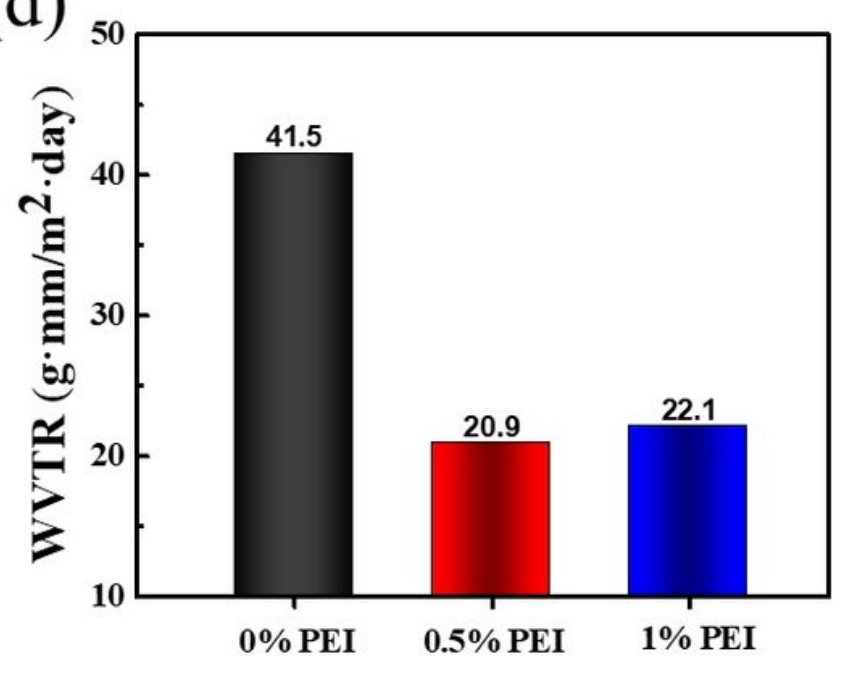

Figure 5

Physical properties of MMT/CNF nanocomposite film with different dosages of PEl. (a) stress-strain curves, (b) toughness, (c) light transmission, and (d)WVTR. Note that the addition of monolayer MMTs is $30 \mathrm{wt} \%$. 
(a)
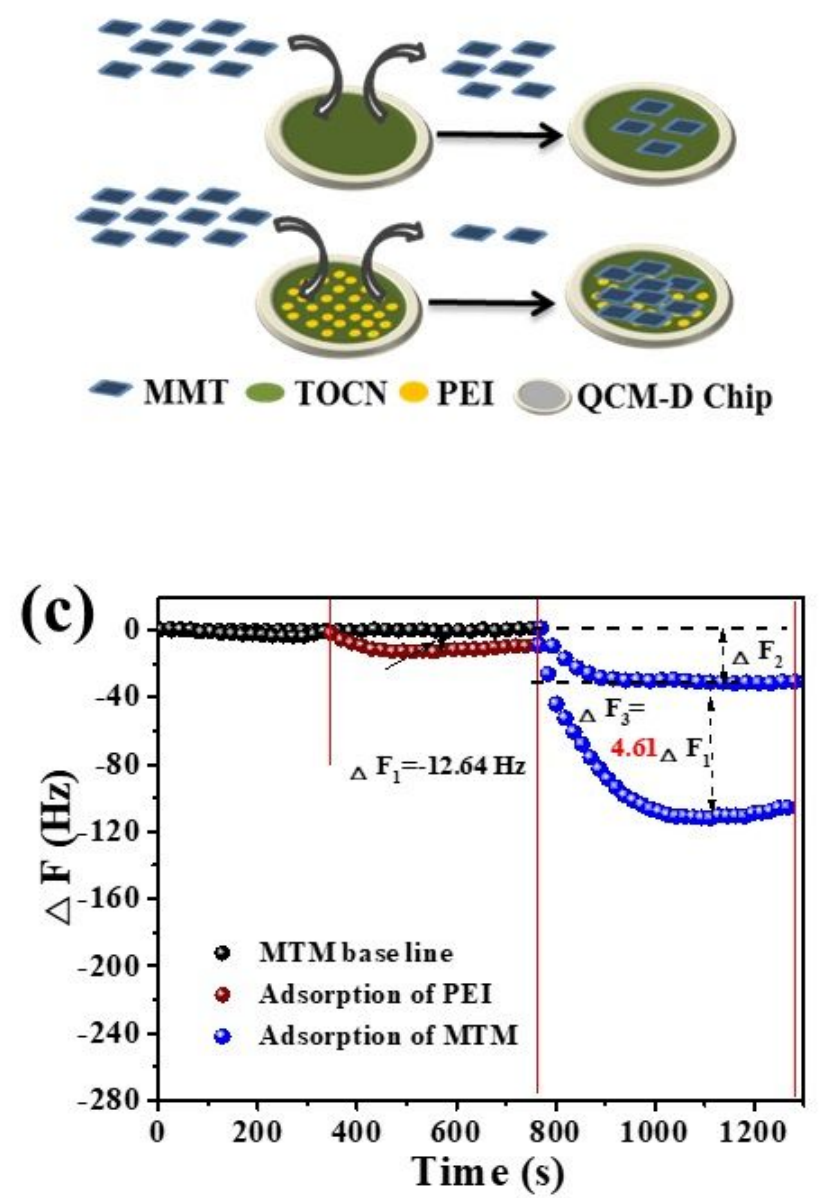

(e)

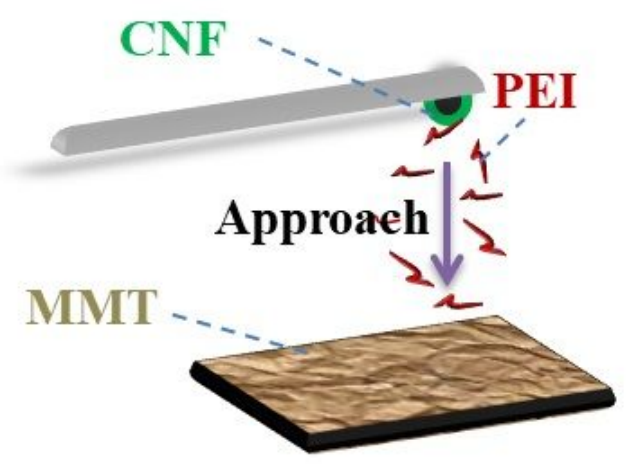

(b)

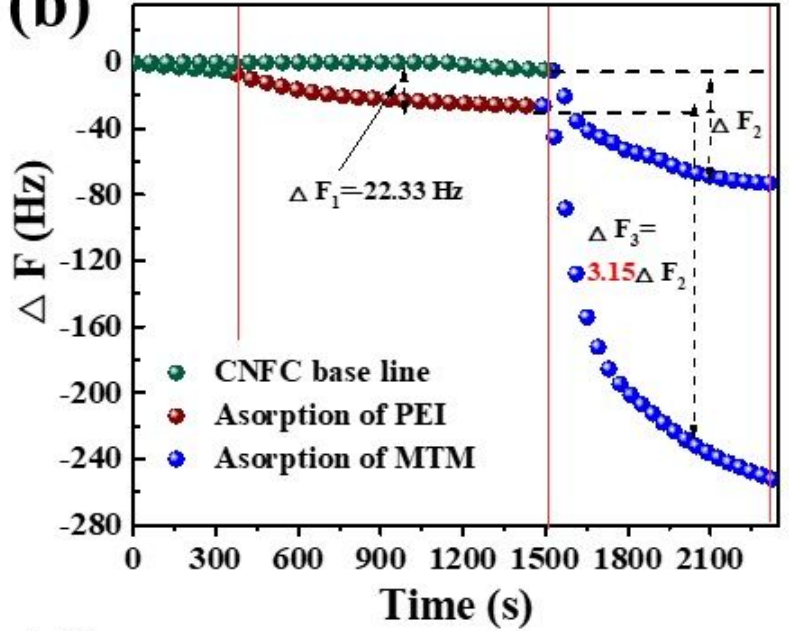

(d)

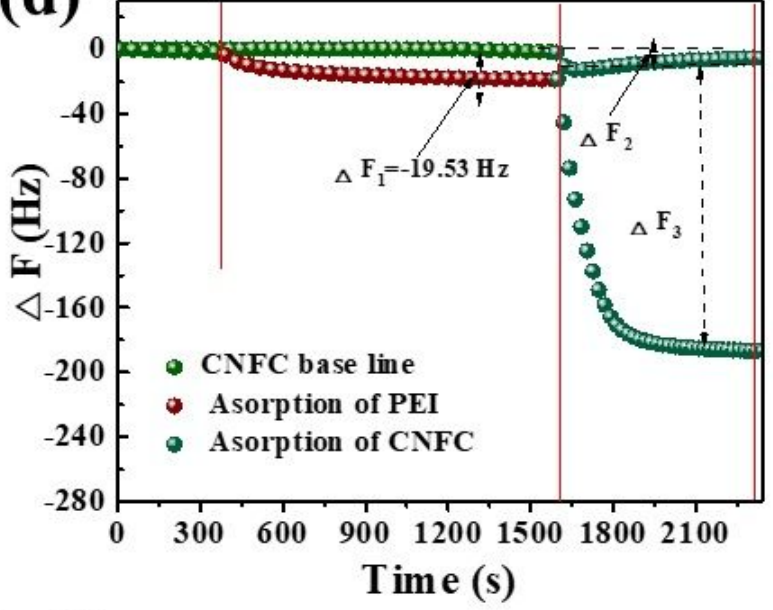

(f)

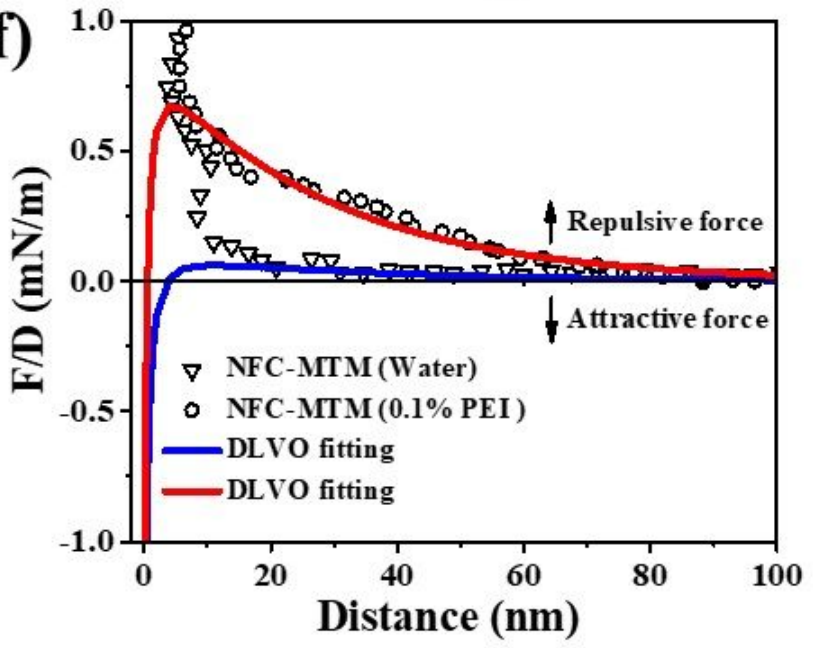

Figure 6

(a) Schematic illustration of the interfacial interactions between MMT and CNF as well as between MMT and PEl-adsorbed CNF by QCM-D analysis. (b) Adsorption behaviors between monolayer MMTs and CNFs (b), between monolayer MMTs (c), as well as between CNFs (d) with/without PEl. (e) Schematic drawing (not to scale) of AFM force measurement. (f) AFM force-distance curves between MMT and CNF in water and in $0.1 \% \mathrm{PEl}$ solution. 
(a)
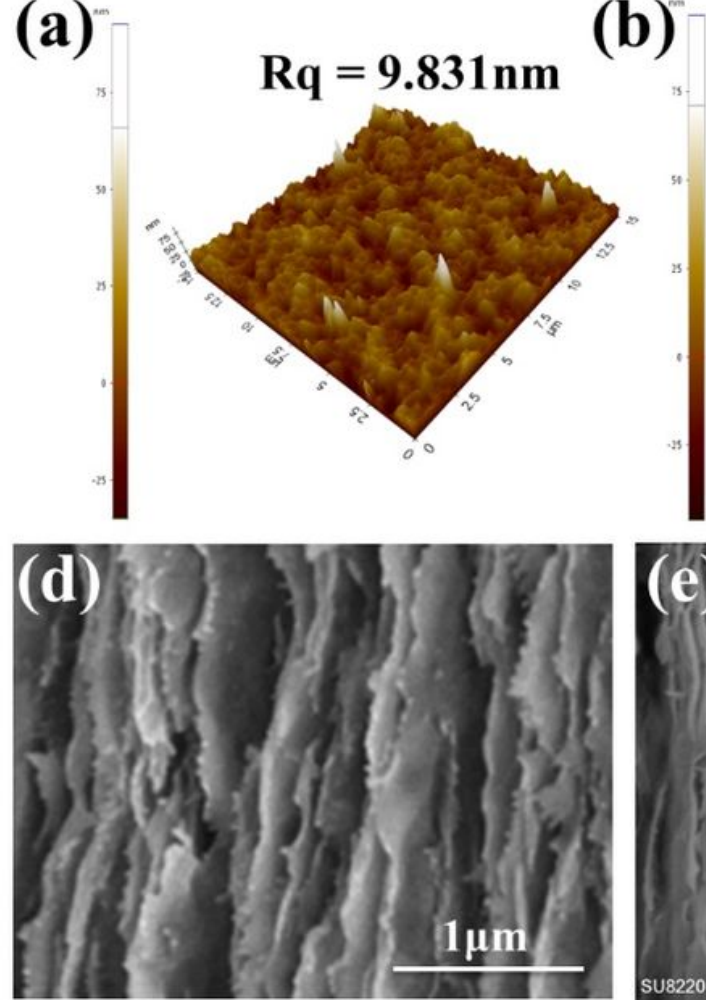

(b)
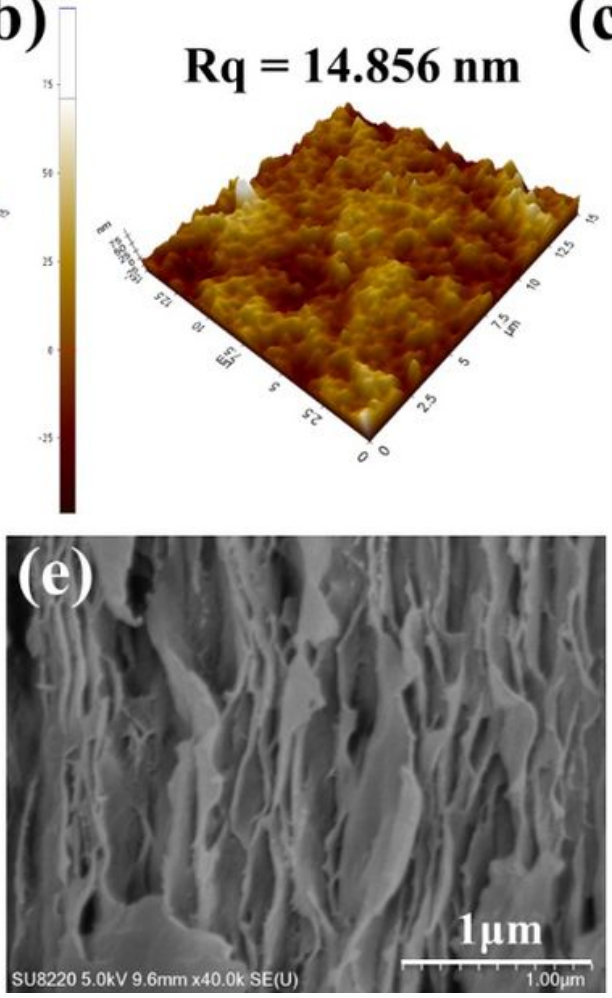

(c)
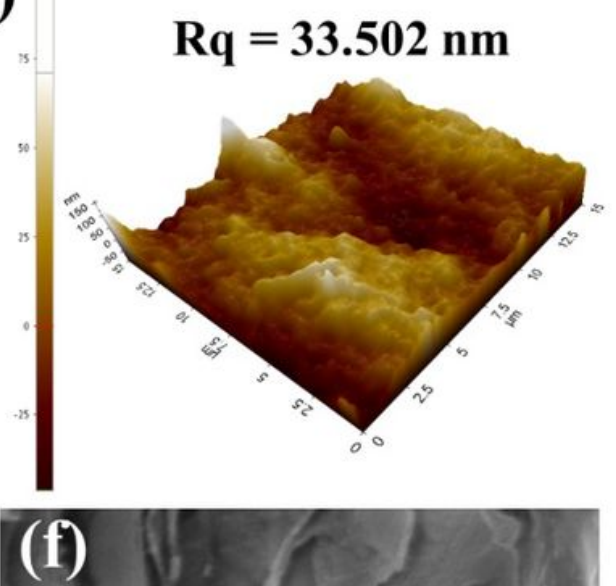

Figure 7

AFM height images of MMT/CNF nanocomposite film with PEI dosages of (a) $0 w t \%$, (b) $0.5 w t \%$, and (c) $1 \mathrm{wt} \%$. Cross-sectional SEM images of MMT/CNF nanocomposite film with PEI dosages of (d) $0 \mathrm{wt} \%$, (e) $0.5 \mathrm{wt} \%$, and (f) $1 \mathrm{wt} \%$.

\section{Supplementary Files}

This is a list of supplementary files associated with this preprint. Click to download.

- renamed1837b.docx

- Graphicalabstract.jpg 